\title{
Les droits linguistiques et la Charte : vingt-cinq années de progression vers l'égalité? Une perspective de l'Ouest canadien
}

\section{Michel Doucet*}

\section{Introduction : Les droits linguistiques dans l'Ouest canadien avant la Charte}

Lors de la Confédération en 1867 certains droits linguistiques seront constitutionnalisés. Larticle 133 de la Loi constitutionnelle de 1867 prévoit un bilinguisme en matière législative et judiciaire, mais seulement en ce qui concerne la province de Québec et le Parlement fédéral ${ }^{1}$. La seule autre province qui ait à l'origine bénéficié de garanties constitutionnelles en matière de droits linguistiques est le Manitoba, aux termes de l'article 23 de la Loi de 1870 sur le Manitoba ${ }^{2}$.

À l'origine, la Saskatchewan et l'Alberta faisaient partie des Territoires du Nord-Ouest et avaient, par conséquent, hérité du dispositif juridique fédéral instauré par l'article 133 de la Loi constitutionnelle de 1867. Les Territoires du Nord-Ouest relevaient de l'article 110 de la Loi sur les Territoires du Nord-Ouest, disposition analogue à l'article $133^{3}$. Après 1892 , cependant les Territoires du Nord-Ouest ne tinrent aucun compte des obligations qui leur incombaient en matière linguistique. La Saskatchewan et l'Alberta, elles aussi, méconnurent, après leur création en 1905, les obligations auxquelles elles étaient tenues dans le domaine. Dans l'arrêt $R$. c. Mercure ${ }^{4}$, la Cour suprême du Canada confirmera cependant que les droits linguistiques énoncés à l'article 110 de la Loi sur les Territoires $d u$ Nord-Ouest continuaient à s'appliquer en Saskatchewan et en Alberta, tout en jugeant que ces droits n'étaient pas ancrés dans la Constitution. Les deux provinces adoptèrent par la suite des lois abrogeant à peu près toutes les garanties linguistiques inscrites dans la Loi sur les Territoires du Nord-Ouest.

En 1982, la Charte canadienne des droits et libertés ${ }^{5}$ est proclamée. Dans ses articles 16 à 23, la Charte offre des garanties linguistiques importantes. La Charte ajoute le NouveauBrunswick au nombre des provinces bilingues et vient renforcer et ajouter aux droits garantis par l'article 133. Pour sa part, l'article 23 de la Charte consacre, dans chacune des provinces et territoires, le droit à l'instruction dans la langue de la minoritéb.

Dans la présentation qui suit, nous tenterons de dégager l'apport des provinces de l'ouest dans le développement de la jurisprudence qui aura permis l'interprétation des garanties linguistiques prévues dans la Charte.

\section{La jurisprudence avant la Charte}

Avant l'adoption de la Charte, la jurisprudence en matière de droits linguistiques était rare. Il y avait bien eu quelques arrêts de la Cour suprême du Canada portant sur l'interprétation des articles 133 de la Loi constitutionnelle de $1867^{7}$, mais règle générale ces décisions se concentraient essentiellement sur des attaques con- 
tre la Loi 101 adoptée par le gouvernement québécois. Dans l'Ouest canadien, cette jurisprudence était rare, pour ne pas dire inexistante.

Pourtant, deux décisions des tribunaux manitobains avaient, vers la fin du XIXe siècle et au début du XXe siècle, reconnu le caractère inaliénable de l'article 23, mais ces deux décisions avaient tout simplement été ignorées par les instances politiques. En effet, en 1892, dans l'affaire Pellant c. Hébert ${ }^{8}$, la Cour avait jugé inconstitutionnelle la Act to Provide that the English Language Shall Be the Official Language of the Province of Manitoba ${ }^{9}$, estimant que le Manitoba n'avait pas la compétence voulue pour modifier ou abroger les garanties linguistiques inscrites à l'article 23. Le Manitoba ne tint cependant aucun compte de cette décision et la pratique de l'unilinguisme s'est poursuivie. En 1909, appelée à se prononcer dans le cadre de l'affaire Bertrand c. Dussault, la Cour de comté de St-Boniface dans un jugement non publié rendu le 30 juin 1909, reproduit dans dans $R e$ Forest and Registrar of Court of Appeal of Manitob $a^{10}$, déclare à nouveau inconstitutionnelle la loi faisant de l'anglais la seule langue officielle de la province. Encore une fois, les autorités provinciales ne tinrent aucun compte de la décision du tribunal.

Ce n'est qu'en 1979, dans l'arrêt Procureur général du Manitoba c. Forest ${ }^{11}$, que la Cour suprême sera appelée à interpréter l’article 23 de la Loi de 1870 sur le Manitoba. Dans cette affaire, la Cour avait à répondre à la question à savoir si la législature du Manitoba n'outrepassait pas ses compétences en adoptant ses lois uniquement en anglais. Il y avait, aux yeux de la Cour, un conflit évident entre la loi provinciale de 1890 faisant de l'anglais la seule langue officielle de la province et l'article 23 de la Loi de 1870 sur le Manitoba. La Cour conclut que la province du Manitoba pouvait modifier sa Constitution, mais qu'elle n'avait pas l'autorité pour modifier les droits linguistiques reconnus par l'article 23. La Cour a assimilé l'article 23 à l'article 133 de la Loi constitutionnelle de 1867, partie intégrante de la Constitution du Canada qui, à ce titre ne saurait être modifiée unilatéralement par une province.

Cette décision faisait de la province du
Manitoba, au moins sur le plan législatif et judiciaire, une province bilingue au même titre que la province de Québec et que le Parlement fédéral.

\section{La jurisprudence après la Charte}

(a) La contribution de l'Ouest aux décisions ayant permis d'établir les principes d'interprétation de la Charte.

Même si elle ne porte pas sur la Charte, la décision dans l'affaire R. c. Mercure ${ }^{12}$ demeure, malgré tout, incontournable. Dans cette affaire, André Mercure, un prêtre fransaskois, avait été accusé d'excès de vitesse en contravention d'une loi de la province de la Saskatchewan. Devant la Cour provinciale, son avocat avait demandé le report de l'affaire, en attendant que le greffier de l'Assemblée législative de la province puisse produire la disposition en cause dans sa version française. Il demandait également à la Cour de pouvoir enregistrer son plaidoyer en français et de subir son procès dans cette langue. En première instance, le juge Deshaye estima que l'obligation fixée par l'article 110 de la Loi sur les Territoires $d u$ Nord-Ouest en matière de publication des lois ne s'imposait qu'à l'Assemblée territoriale, et non à la législature de la Saskatchewan $^{13}$. La Cour d'appel rejeta le pourvoi du père Mercure ${ }^{14}$. La Cour suprême accepta d'entendre le pourvoi.

Le juge La Forest a rendu le jugement pour la majorité de la Cour. Il estima que la question qui était soumise à la Cour pouvait être tranchée au vu des principes normalement applicables en matière d'interprétation des lois. L'ensemble des parties ayant insisté sur l'histoire législative des dispositions en cause, il se livra également à un long et intéressant exposé historique de l'origine de l'article $110^{15}$.

Dans cette décision, le juge La Forest insista sur l'importance que revêtent les droits linguistiques $^{16}$. En ce qui concerne l'article 110, la Cour conclut que cette mesure législative était toujours en vigueur et que les lois de la Saskatchewan, et implicitement celles de l'Alberta, devaient être promulguées, imprimées et publiées en français et en anglais. Le juge La Forest 
ajoute qu'il serait faux de voir dans l'article 110 qu'une mesure transitoire et il rappelle qu'une telle disposition demeure en vigueur jusqu'à son abrogation.

Bien que la Cour ait conclu que l'article 110 était toujours en vigueur lors de la création des provinces de la Saskatchewan et de l'Alberta, elle ajoute qu'il n'a pas été constitutionnalisé, comme ce fut le cas pour l'article 23 de Loi de 1870 sur le Manitoba. Puisqu'elle n'est pas une disposition constitutionnelle, elle peut donc être modifiée ou abrogée par une simple $\operatorname{loi}^{17}$.

Les lois promulguées en anglais seulement par la législature ont été déclarées invalides et celle-ci fut tenue de prendre les mesures nécessaires pour rectifier à ce manquement dans un délai raisonnable. Selon le juge La Forest, la législature pouvait procéder à la traduction des lois et, ensuite, les promulguer, les faire imprimer et publier dans les deux langues. Elle pouvait aussi recourir à "l'expédient manifeste, voire même ironique » d'adopter une loi bilingue supprimant la restriction imposée par l'article 110 en matière de mode et de forme, puis déclarer valides rétroactivement, toutes les lois provinciales malgré leur promulgation, leur impression et leur publication dans leur seule version anglaise ${ }^{18}$. C'est d'ailleurs cet expédient « ironique » qu'utiliseront l'Alberta et la Saskatchewan pour se débarrasser de l'irritant que constituait l'article 110.

L'arrêt de la Cour suprême n'échappe certainement pas à la critique. En effet, la conclusion de la Cour dans cette affaire confirme que ce qui pour les Franco-manitobains est immuable (l'article 23 de la Loi de 1870 sur le Manitoba) peut, dans le cas des provinces de l'Alberta et de la Saskatchewan, être abrogé par une simple loi provinciale. Cette conclusion de la Cour est lourde de conséquences pour les communautés francophones du Canada, puisqu'elle semble reconnaître que les gouvernements provinciaux peuvent abroger sans problème les droits linguistiques législatifs de ces provinces. Ainsi, seules les provinces du Nouveau-Brunswick, du Québec et du Manitoba seraient à l'abri de telles modifications puisqu'elles ont des droits linguistiques constitutionnalisés.
Cette conclusion de la Cour suprême nous semble cependant contraire au principe de la progression vers l'égalité reconnue au paragraphe 16(3) de la Charte, ainsi qu'au principe constitutionnel non écrit de la protection des minorités reconnu dans le Renvoi relatif à la sécession du Québec ${ }^{19}$. Nous reviendrons sur ces questions un peu plus loin dans notre exposé.

Revenons maintenant aux décisions portant spécifiquement sur la Charte. Une décision importante lorsque vient le temps de parler de principes d'interprétation des droits linguistiques de la Charte est, sans contredit, le Renvoi: Droits linguistiques au Manitoba ${ }^{20}$. Cette décision pose comme principe que l'article 23 de la Loi de 1870 sur le Manitoba est une manifestation d'un droit général pour tous les Manitobains, francophones comme anglophones, à "l'accès égal au corps législatif, aux lois et aux tribunaux ${ }^{21} »$. De plus, la Cour ajoute que les droits linguistiques sont fondés sur la dignité humaine :

L'importance des droits en matière linguistique est fondée sur le rôle essentiel que joue la langue dans l'existence, le développement et la dignité de l'être humain. C'est par le langage que nous pouvons former des concepts, structurer et ordonner le monde autour de nous. Le langage constitue le pont entre l'isolement et la collectivité, qui permet aux êtres humains de délimiter les droits et obligations qu'ils ont les uns envers les autres, et ainsi, de vivre en société22.

Malheureusement, cette approche généreuse quant à l'interprétation des droits linguistiques sera oubliée pendant un certain temps, à la suite de trois décisions de la Cour suprême du Canada rendues le même jour, et dont une provenait du Manitoba. En effet, dans la trilogie Société des Acadiens du Nouveau-Brunswick c. Association of Parents for Fairness in Education ${ }^{23}$, MacDonald c. Ville de Montréal ${ }^{24}$, et Bilodeau c. Procureur général du Manitoba ${ }^{25}$, la Cour suprême adopte une position selon laquelle les droits linguistiques sont le fruit de compromis politiques et qu'en conséquence, ils doivent être interprétés restrictivement. L'article 19 de la Charte, l'article 133 de la Loi constitutionnelle de 1867 et l'article 23 de la Loi de 1870 sur le Manitoba sont interprétés de telle manière que 
le droit de parler l'une ou l'autre des langues officielles devant une cour de justice n'inclut pas le droit d'être compris par le juge directement dans cette langue.

Or, les décisions qui suivront, dont plusieurs tirent leur origine de litige ayant pris naissance dans l'Ouest, viendront atténuer le caractère restrictif de la trilogie. Ainsi dans $R$ c. Mercure, la majorité de la Cour relie l'interprétation des droits linguistiques à leur objet, en reconnaissant qu'ils sont un enjeu national important et qu'ils participent des droits fondamentaux de la personne ${ }^{26}$.

Dans Mahe c. Alberta ${ }^{27}$, écartant l'approche restrictive de la trilogie, la Cour suprême conclut que l'article 23 de la Charte doit s'interpréter selon son objet :

L’objet général de l'art. 23 est clair : il vise à maintenir les deux langues officielles du Canada ainsi que les cultures qu'elles représentent et à favoriser l'épanouissement de chacune de ces langues, dans la mesure du possible, dans les provinces où elle n'est pas parlée par la majoritée ${ }^{28}$.

Elle insiste aussi sur le lien étroit qui existe entre langue et culture :

\begin{abstract}
Mon allusion à la culture est importante, car il est de faits que toute garantie générale de droits linguistiques, surtout dans le domaine de l'éducation, est indissociable d'une préoccupation à l'égard de la culture véhiculée par la langue en question. Une langue est plus qu'un simple moyen de communication; elle fait partie intégrante de l'identité et de la culture du peuple qui la parle. C'est le moyen par lequel les individus se comprennent eux-mêmes et comprennent le milieu dans lequel ils vivent ${ }^{29}$.
\end{abstract}

Arrive ensuite l'arrêt $R \quad$ c. Beaulac ${ }^{30}$, qui prend origine en Colombie-Britannique et qui porte sur l'interprétation de l'article $530 \mathrm{du}$ Code criminel canadien, lequel garantit à un accusé le droit à un procès dans l'une ou l'autre des langues officielles ${ }^{31}$. Dans cette décision, la Cour suprême abandonne de manière non équivoque la théorie du "compromis politique» énoncée dans la trilogie au profit d'une approche téléologique fondée sur l'objet des droits linguistiques :
Les droits linguistiques doivent dans tous les cas être interprétés en fonction de leur objet, de façon compatible avec le maintien et l'épanouissement des collectivités de langue officielle au Canada; voir Renvoi relatif à la Loi sur les écoles publiques (Man.), précité, à la p. 850. Dans la mesure où l'arrêt Société des Acadiens du Nouveau-Brunswick, précité, aux pp. 579 et 580, préconise une interprétation restrictive des droits linguistiques, il doit être écarté ${ }^{32}$.

La décision dans l'affaire Beaulac marquera la renaissance d'une interprétation libérale des droits linguistiques. Elle sera suivie d'autres décisions qui viendront donner une nouvelle impulsion aux droits linguistiques ${ }^{33}$.

\section{Les articles 16 à 20 de la Charte}

Puisque ces dispositions ne s'appliquent pas spécifiquement aux provinces de l'ouest, il y a peu de jurisprudence provenant de cellesci portant sur leur interprétation. D’ailleurs, même au niveau canadien et néo-brunswickois, la jurisprudence portant sur ces dispositions fait grandement défaut.

Toutefois, certaines décisions méritent que nous nous y attardions. Nous avons plus haut fait référence à la décision dans l'affaire Mercure dans laquelle la Cour suprême avait jugé qu'il n'y avait pas d'obligation linguistique constitutionnelle dans le processus législatif en Saskatchewan et, indirectement en Alberta, parce que l'article 110 de la Loi sur les Territoires $d u$ Nord-Ouest n'avait pas été enchâssé dans la Constitution. En 1995, un autre fransaskois, M. René Rottiers, a demandé aux tribunaux de préciser si la législature de la Saskatchewan n'avait pas une certaine obligation de traduire des lois provinciales importantes ou d'intérêt public en Saskatchewan, mais la Cour d'appel a rejeté cet $\operatorname{argument}^{34}$.

Cependant, depuis que la Cour suprême du Canada a rendu son opinion dans le Renvoi sur la sécession du Québec, la question du réexamen de la validité constitutionnelle de l'article 110 est à nouveau soulevée, cette fois en Alberta. Le 2 juillet 2008, l'honorable juge Leo Wenden, de la Cour provinciale de l'Alberta rendait un juge- 
ment dans une affaire impliquant Gilles Caron, un francophone résidant en Alberta, qui était accusé d'avoir contrevenu au paragraphe 34(2) de la Use of the Highways and Rules of the Road Regulations. Dans ce jugement la Cour provinciale déclarait inopérants, en ce qui concerne l'accusé, Gilles Caron, l'article 4 de la Languages Act, R.S.A. et le paragraphe 34(2) des Use of the Highways and Rules of the Road Regulations, parce que ces dispositions n'ont pas été publiées en français. Dans ses motifs, la Cour provinciale conclut également que la Constitution protège, en Alberta, le statut et l'usage du français :

- dans la législature de l'Alberta et relativement aux lois adoptées en Alberta ;

- dans le système judiciaire albertain; et

- dans le domaine des services offerts par le gouvernement de l'Alberta ${ }^{35}$ ?

La remise en question de la décision de la Cour suprême dans l'arrêt Mercure est d'autant plus pertinente si nous prenons en considération le principe de la progression vers l'égalité reconnue au paragraphe 16(3) de la Charte. Ce paragraphe exprime l'objectif constitutionnel suivant : faire progresser l'égalité réelle des deux langues officielles au Canada ${ }^{36}$. Cette disposition, interprétée à la lumière du principe constitutionnel non écrit de la protection des minorités, aurait dû empêcher le gouvernement de l'Alberta de prendre des décisions ayant pour effet le recul dans l'égalité de la langue française et de la langue anglaise dans cette province. S'il est exact que l'Alberta n'a pas d'obligations constitutionnelles explicites découlant des articles 17 à 22 de la Charte, il importe d'ajouter qu'elle demeure liée par le paragraphe 16(3) et par le principe qui en découle.

Les deux langues officielles ont reçu au Canada un statut constitutionnel par l'inscription dans la Charte des droits linguistiques. Mais cette inscription est incomplète et participe d'un processus plus large toujours inachevé. D'ailleurs, la conclusion de la Cour suprême dans le Renvoi relatifà la sécession du Québec en est une confirmation. En effet, le texte du paragraphe 16(3) révèle qu'il vise beaucoup plus que d'étendre le statut et l'usage des langues officiel- les inscrit à l'article 16. Il est juste que ce paragraphe vise à codifier le principe de l'arrêt Jones c. Procureur général du Nouveau-Brunswick ${ }^{37}$, à savoir que les dispositions constitutionnelles de la Charte sont un seuil, et non un plafond. Le Parlement et les législatures provinciales, dans leurs sphères de compétence respectives, peuvent ajouter à ces droits. Or, ce paragraphe renferme également un principe de "progression" vers l'égalité de statut ou d'usage des langues officielles.

Pour appuyer nos prétentions, nous appuyons sur l'opinion dissidente de la juge Wilson, qui déclarait dans l'arrêt Société des Acadiens du Nouveau-Brunswick:

\begin{abstract}
Toutefois, il ressort clairement du par. 16(3) que ces conséquences représentent le but visé plutôt que la réalité actuelle; il s'agit de quelque chose dont le Parlement et les législatures doivent «favoriser la progression». Cela semblerait conforme à l'esprit de l'arrêt Jones $c$. Procureur général du Nouveau-Brunswick [...], savoir que les législatures ne peuvent déroger à des droits déjà accordés, mais elles peuvent en étendre la portée. À condition que la législation "favorise la progression vers l'égalité de statut des deux langues officielles, les cours n'y toucheront pas; dans l'hypothèse contraire, elle ne résistera pas à l'examen judiciaire. J'estime toutefois qu'aucun droit à un redressement ne découle inévitablement du fait que le but n'ait pas encore été atteint à un moment donné. J'abonde dans le sens de ceux qui voient dans l'art. 16 un principe de croissance ou de développement, une progression vers un objectif ultime. La question, selon moi, sera donc toujours de savoir où nous en sommes présentement dans notre cheminement vers le bilinguisme et si la conduite attaquée peut être considérée comme appropriée à ce stade de l'évolution. Dans l'affirmative, même si la conduite en question ne reflète pas la pleine égalité de statut et l'égalité quant aux droits à l'usage des langues officielles, elle ne sera pas contraire à l'esprit de l'art. 16 (emphase par l'auteur) ${ }^{38}$.
\end{abstract}

Cette opinion dissidente rejoint les propos du juge Bastarache dans l'arrêt $R$. c. Beaulac, lorsqu'il affirme :

Je conviens que l'existence d'un compromis politique n'a aucune incidence sur l'étendue des droits linguistiques. L'idée que le par. 16(3) 
de la Charte qui a officialisé la notion de progression vers l'égalité des langues officielles du Canada exprimée dans l'arrêt Jones, précité, limite la portée du par. 16(1) doit également être rejetée. Ce paragraphe confirme l'égalité réelle des droits linguistiques constitutionnels qui existent à un moment donné (emphase par l'auteur ${ }^{39}$.

De ce passage de Beaulac, nous retenons :

1. que le paragraphe 16(3) officialise la notion de "progression " vers l'égalité des langues officielles au Canada; et

2. qu'il confirme l'égalité réelle des droits linguistiques constitutionnels qui existent à un moment donné.

Ainsi, il devrait normalement s'ensuivre qu'un recul dans la "progression» vers l' " égalité réelle» des deux langues officielles nuira à cette « égalité réelle » des droits linguistiques constitutionnels qui existent à un moment donné. En conséquence, l'Alberta ne peut, par l'adoption de sa Loi linguistique ${ }^{40}$, déroger aux droits linguistiques dont bénéficiait la communauté linguistique francophone de cette province en raison de l'article 110 de la Loi des Territoires du Nord-Ouest. C'est à la lumière de ces arguments qu'un tribunal devrait trancher la constitutionnalité de la Loi linguistique et faire une nouvelle lecture de la décision dans l'affaire Mercure où ces arguments n'avaient pas été soulevés.

Il est important également de rappeler que les propos du juge La Forest dans l'arrêt Mercure, concernant les différents moyens mis à la disposition des gouvernements de l'Alberta et de la Saskatchewan pour répondre au problème que soulève pour eux l'article 110 , faisaient parties d'une remarque incidente. Une lecture attentive des motifs révèle, à notre avis, que cet élément de son analyse ne constituait pas un maillon essentiel de son raisonnement sur la question qui lui était posée et que, par conséquent, elle ne fait pas partie de son ratio decidendi. Or, si tel est le cas, la question de la constitutionnalité de la Loi linguistique n'est pas res judicata et demeure entière. En d'autres mots, le tribunal saisi de l'affaire Caron est pleinement compétent pour juger de la constitutionnalité de la Loi linguistique. Nous soutenons que le pouvoir du gouvernement d'abroger l'article 110 ne lui conférait aucunement le droit d'enlever, sans justification légitime, l'essentiel des droits qui étaient reconnus à l'article 110. À tout le moins, avant de prendre cette décision, il avait l'obligation d'évaluer quel impact celle-ci aurait sur la communauté minoritaire de langue officielle. La question, selon nous, est donc de savoir où nous en sommes actuellement dans notre cheminement vers l'égalité des langues officielles en Alberta, et si la conduite attaquée peut être considérée comme appropriée à ce stade de cette évolution. À notre avis, le gouvernement albertain n'a jamais fait cette preuve et ne peut donc justifier l'abolition de l'article 110.

Dans une autre affaire qui touche cette fois directement aux dispositions de la Charte, la Cour d'appel des Territoires du Nord-Ouest (T.N.-O.) est saisie de la question de savoir si les institutions des Territoires du Nord-Ouest sont des « institutions du Parlement et du gouvernement du Canada » au sens des articles 16 et 20 de la Charte et, dans l'affirmative, quelle est la responsabilité du gouvernement fédéral à cet égard. ${ }^{41}$.

Bien quelle ne porte pas sur une disposition de la Charte, j'attire également votre attention sur la décision récente de la Cour d'appel du Yukon dans l'arrêt Kilrich Industries Ltd. c. Halotier ${ }^{42}$.

\section{L'article 23 et le droit à l'éducation dans la langue de la minorité}

L'acquis le plus important pour les communautés minoritaires de langue officielle lors de l'adoption de la Charte est sans contredit l'article 23. Cet article prévoit essentiellement le droit pour les parents appartenant à une minorité linguistique de langue officielle dans la province et les territoires où ils résident de faire instruire leurs enfants dans cette langue aux niveaux primaire et secondaire ${ }^{43}$. Toutefois, ce droit est garanti uniquement lorsque «le nombre d'enfants le justifie ». Par ailleurs, si le nombre le justifie, ce droit à l'instruction comprend également le droit à des " établissements d'enseignement de la minorité linguistique » et éventuellement un 
« droit de gestion».

Pour obtenir ce droit, les parents doivent démontrer qu'ils appartiennent à l'une des trois catégories de titulaires prévues par l'article 23. La première catégorie comprend les personnes dont la première langue apprise et encore comprise est celle de la minorité francophone ou anglophone. La deuxième catégorie concerne les parents qui ont reçu, au Canada, leur instruction primaire dans la langue de la minorité de la province où ils résident. Enfin, la troisième catégorie prévue par le paragraphe 23(2) dépend de la langue d'instruction de l'enfant ${ }^{44}$.

L'article 23 a donné lieu à plusieurs contestations judiciaires, lesquelles ont contribué à clarifier, d'une part, la nature et la portée des droits linguistiques des parents et, d'autre part, les obligations incombant aux gouvernements provinciaux pour en assurer la mise en œuvre. Plusieurs de ces décisions tirent leur origine de contestations judiciaires initiées dans les provinces de l'ouest.

Dans l'arrêt de principe, Mahe c. Alberta, la Cour suprême du Canada affirme que l'article 23 a un caractère réparateur ${ }^{45}$. Elle ajoute également qu'une interprétation restrictive de cette disposition serait inappropriée. Selon la Cour, l'article 23 est destiné à remédier, à l'échelle nationale, à l'érosion progressive des minorités parlant l'une ou l'autre des langues officielles. En outre, la Cour a reconnu que, de par leur nature même, les droits garantis par l'article 23 imposent aux gouvernements des obligations positives.

En ce qui concerne le droit à l'instruction, les tribunaux précisent que l'article 23 garantit la possibilité de recevoir une instruction " dans le cadre d'un programme adapté aux besoins de la minorité et reflétant ses valeurs et sa culture ${ }^{46}$ » et a ajouté que « les élèves de langue française devraient vivre en français dans la cour de récréation, à l'occasion des activités hors programme ainsi que dans les classes ${ }^{47}$ ».

Dans l'affaire Mahe, la Cour suprême avait également ajouté que la disposition accordait aux parents le droit de gestion lorsque le nombre d'enfants le justifie. La Cour y a aussi donné plusieurs indications relativement au droit de gestion des parents ayants droit. Elle a pris soin, par exemple, de préciser que cette gestion et ce contrôle sont essentiels au respect de l'objet de l'article 23 de la Charte, qui consiste à « maintenir les deux langues officielles du Canada ainsi que les cultures qu'elles représentent et à favoriser l'épanouissement de chacune de ces langues, dans la mesure du possible, dans les provinces où elle n'est pas parlée par la majoritét ${ }^{2} »$. Elle a ajouté que " cette gestion et ce contrôle [...] sont nécessaires parce que plusieurs questions de gestion en matière d'enseignement (programmes d'études, embauchage et dépenses, par exemple) peuvent avoir des incidences sur les domaines linguistique et culturel ${ }^{49}$ ». Ce qui est essentiel, dira la Cour, c'est que le groupe linguistique minoritaire ait un contrôle sur " les aspects de l'éducation qui concernent ou qui touchent sa langue et sa culture ${ }^{50} »$.

Enfin, la Cour suprême du Canada a interprété l'article 23 de la Charte comme prévoyant un « droit à contenu variable» en ce qui concerne à la fois le droit à l'instruction et le droit de gestion. Ce qui est requis dans chaque cas dépend de ce qui est justifié par le nombre d'enfants, et ce nombre devrait en principe correspondre au nombre de personnes raisonnablement envisageables qui pourrait se prévaloir de ce droit. Ce qui est requis dans chaque cas dépend principalement de considérations pédagogiques et financières. Dans tous les cas, l'instruction dans la langue de la majorité et dans celle de la minorité devra toutefois être de qualité équivalente.

Ainsi, selon le nombre d'élèves en cause, l'article 23 de la Charte peut justifier l'établissement de classes ou la mise en place d'établissements d'enseignement de la minorité linguistique financés par les fonds publics. Quant au degré de gestion ou de contrôle, l'article 23 peut, toujours en fonction du nombre d'enfants, justifier l'existence d'un conseil scolaire de la minorité linguistique.

À la suite du Renvoi relatif à la Loi sur les écoles publiques au Manitoba qui avait établi, comme nous venons de le voir, que le droit aux établissements comprenait celui de recevoir l'enseignement dans des lieux physiques dis- 
tincts, des tribunaux ont reconnu que, dans certaines circonstances, les enfants de la minorité pouvaient partager certains types de locaux avec des enfants de la majorité.

C'est ainsi que la Cour d'appel de la Saskatchewan rendait, dans la décision Conseil scolaire fransaskois de Zenon Park c. Saskatch$e w a n^{51}$, une ordonnance obligeant la construction d'édifices scolaires mobiles pour la minorité à côté d'une école de la majorité et a exigé que cette dernière fournisse à la minorité l'accès au gymnase, aux vestiaires et aux toilettes de même qu'au laboratoire de sciences, à la bibliothèque et à la médiathèque.

Ces décisions montrent la contribution importante que les affaires judiciaires initiées dans les provinces de l'Ouest ont pu avoir sur l'interprétation de l'article 23. Ces décisions ont nécessité l'engagement de justiciables qui ont accepté d'agir afin que la collectivité francophone dans son ensemble puisse profiter des droits qu'on lui avait reconnus, mais que l'on tardait à mettre en œuvre. Ces décisions montrent également la détermination d'avocats qui en utilisant une bonne dose d'imagination et de conviction ont réussi à convaincre les tribunaux que les droits linguistiques n'étaient pas inertes, mais qu'ils exigeaient une interprétation généreuse si l'on voulait qu'ils réalisent l'objectif qui est le leur.

\section{Conclusion}

Les provinces de l'Ouest canadien ont été une terre fertile pour l'évolution judiciaire des droits linguistiques reconnus dans la Charte et dans les autres textes constitutionnels. Malgré les aléas de l'histoire qui ne leur ont pas toujours été favorables, les communautés francophones de l'Ouest n'ont pas hésité à utiliser les quelques droits constitutionnels qui leur étaient reconnus afin d'assurer le développement et l'épanouissement de leurs communautés. Acceptant de relever une tâche que plusieurs considéraient comme impossible, ils ont réussi à faire renaître des droits et à faire avancer ceux qui leur avaient été parcimonieusement accordés en 1982, avec l'adoption de la Charte.

\section{Notes}

* Michel Doucet, avocat et professeur titulaire, Faculté de droit, Université de Moncton.

Présenté dans le cadre du colloque portant sur le, $" 25^{\mathrm{e}}$ anniversaire de la Constitution canadienne : perspective de l'Ouest canadien » organisé par la University of Alberta et l'Institut d'études canadiennes, le 24 novembre 2007.

$1 \quad$ Loi constitutionelle de 1867 (R.-U.), 30 \& 31 Vict., c.3, art. 133, reproduit dans L.R.C. 1985, app. II, $\mathrm{n}^{\circ} 5$ (IIJCan).

2 Loi de 1870 sur le Manitoba, S.R.C. 1970, app. II, art. 23.

3 Loi sur les Territoires du Nord-Ouest (L.R., 1985, ch. N-27).

41988 CSC 107, [1988] 1 R.C.S. 234 (IIJCan) [Mercure].

$5 \quad$ Charte canadienne des droits et libertés, partie I de la Loi constitutionelle de 1982, constituant l'annexe B de la Loi de 1982 sur le Canada (R.U.), 1982, c.11 (IIJCan) [Charte].

$6 \quad$ Ibid. à l'article 23.

7 Jones c. Procureur général du Nouveau-Brunswick, [1975] 2 R.C.S. 182; Blaikie c. Procureur général du Québec, [1979] 1 R.C.S. 1011 ; Blaikie c. Procureur général du Québec (no 2), [1981] 1 R.C.S. 312.

8 Cour de comté de St-Boniface, 9 mars 1892, jugement non publié, reproduit (1981) 12 R.G.D. 242.

91890 (Man.), c. 14.

10 (1977), 77 D.L.R. (3d) 445.

11 [1979] 2 R.C.S. 1032.

12 Supra note 4.

13 (1981), 44 Sask. R. 43 (C.P.).

14 (1985), 44 Sask. R. 22 (C.A.).

15 Supra note 4 aux pp. 248-57.

16 Ibid. aux pp. 264 à 270.

17 Ibid à la p. 272.

18 Ibid aux pp. 280-81.

191998 CSC 793, [1998] 2 R.C.S. 217 (IIJCan).

201985 CSC 33, [1985] 1 R.C.S. 721 (IIJCan).

21 Ibid. au para. 31.

22 Ibid. au para. 46.

23 [1986] 1 R.C.S. 549 [Société des Acadiens du Nouveau-Brunswick].

24 [1986] 1 R.C.S. 460. [1986] 1 R.C.S. 449.

Supra note 4. 1990 CSC 133, [1990] 1 R.C.S. 342 (IIJCan). Ibid. au para. 31.

Ibid. au para. 32 .

1999 CSC 684, [1999] 1 R.C.S. 768

(IIJCan)[Beaulac].

31 Code criminel, L.R.C. 1985, c. C-46, article 530. 
32 Supra note 30 au para. 25.

33 Voir entre autres Renvoi sur la sécession du Québec, supra note 19 ; Arsenault-Cameron c. Île-duPrince-Édouard, 2000 CSC 1, [2000] 1 R.C.S. 3 (IIJCan); et Doucet-Boudreau c. Nouvelle-Écosse (Ministre de l'Éducation), 2003 CSC 62, [2003] 3 R.C.S. 3 (IIJCan).

34 R. c. Rottiers, (1995) 134 Sask. R. 153 (C.A.).

35 Voir R. c. Caron, 2008 ABPC 232 (IIJCan), Le dossier a été porté en appel par le Procureur général de l'Alberta et devrait être entendu par la Cour du Banc de la Reine au début de l'année 2009.

36 Supra note 5, article 16(3).

37 Jones, supra note 7.

38 Supra note 23 au para. 140.

39 Supra note 30 au para. 24.

40 Languages Act (French version), R.S.A. 2000, c. L-6 (français) [Loi linguistique].

41 Fédération franco-ténoise c . Canada (Procureur général), 2006 NWTSC 20, modifiée dans 2008 NWTCA 26

$42 \quad 2007$ YKCA 12.

43 Supra note 5 à l'article 23.

44 Ibid.

45 Supra note 27.

46 Reference re Education Act (Ont.) and Minority Language Rights (1984), 47 R.J.O. (2d) 1, par. 39 et 40 (C.A.).

47 Renvoi relative à la Loi sur les écoles publiques par. 79(3), (4) et (7), [1993] 1. R.C.S. 839, p. 854 et 855, citant Renvoi relatif à la Loi sur les écoles publiques par. 79(3), (4) et (7), (1990), 64 Man. R, (2d) 1 (C.A.).

48 Supra note 27 au para. 31.

$49 \quad$ Ibid au para. 51.

$50 \quad$ Ibid au para. 57.

51 [1999] 3 W.W.R. 743, confirmé par [1999] 12 W.W.R. 742. 